\title{
Construction of Sources for Majumdar-Papapetrou Spacetimes
}

\author{
Victor Varela \\ Escuela de Física, Facultad de Ciencias, \\ Universidad Central de Venezuela, \\ Caracas, Venezuela \\ E-mail: vvarela@fisica.ciens.ucv.ve
}

\begin{abstract}
We study Majumdar-Papapetrou solutions for the 3+1 Einstein-Maxwell equations, with charged dust acting as the external source for the fields. The spherically symmetric solution of Gürses is considered in detail. We introduce new parameters that simplify the construction of class $C^{1}$, singularity-free geometries. The arising sources are bounded or unbounded, and the redshift of light signals allows an observer at spatial infinity to distinguish these cases. We find out an interesting affinity between the conformastatic metric and some homothetic, matter and Ricci collineations. The associated non-Noetherian symmetries provide us with distinctive solutions that can be used to construct non-singular sources for Majumdar-Papapetrou spacetimes.
\end{abstract}

\section{Introduction}

Majumdar [1] and Papapetrou [2] showed that assuming a static spacetime metric with the conformastatic [3] form

$$
d s^{2}=-V^{2} d t^{2}+\frac{1}{V^{2}} d \vec{x} \cdot d \vec{x}
$$

where $V=V\left(x^{1}, x^{2}, x^{3}\right)$, the task of finding solutions for the Einstein-Maxwell equations can be greatly simplified. In fact, assuming a linear relationship between the time-like component of the electromagnetic potential and $V$, they found that $\frac{1}{V}$ is harmonic, i.e. it is a solution of the Laplace equation. Therefore, the problem of solving the coupled field equations reduces to solving the Laplace equation in a threedimensional, Euclidean space which is called the "background space". Solutions with point-like singularities distributed in a bounded region of the background space imply asymptotically flat spacetimes and have the simple form

$$
\frac{1}{V}=1+\sum_{i=1}^{N} \frac{m_{i}}{r_{i}},
$$

where

$$
r_{i}=\sqrt{\left(x-x_{i}\right)^{2}+\left(y-y_{i}\right)^{2}+\left(z-z_{i}\right)^{2}} .
$$


A complete analysis of the singularities of these electrovac solutions was presented by Hartle and Hawking [4] who showed that in a static system of electric charges of the same sign, all the genuine spacetime singularities are contained within event horizons. This class of electrovac solutions describe systems of charged black holes in equlibrium under their mutual gravitational and electrical interactions.

The singularities of the Majumdar-Papapetrou (MP) solution (2) can be avoided in principle if we consider extended sources for the electric and gravitational fields e.g. charged fluids. Das [5] discussed the extension of the MP procedure to the case in which the fluid is charged dust. As a result, $\frac{1}{V}$ comes out as the solution of a potential equation which is generally non-linear and additional information about the energy density of the fluid, $\rho$, must be provided in order to obtain particular solutions.

Gürses [6] showed how a particular choice for the functional $\rho=\rho(V)$ makes the potential equation linear and leads to simple analytical, internal solutions that can be matched with the external solution (2) at the boundary of the charged source, without symmetry restrictions. Other choices for $\rho$ were considered by Varela [7], who discussed charged dust solutions in the non-linear case of this equation. Ida [8] studied solutions with charged perfect fluids, and Cho et al. 9 9 generalised this theory with the inclusion of a dilaton field.

A different approach was presented by Bonnor and Wickramasuriya [10, 11] and Bonnor [12], who constructed non-singular charged dust sources with spherical and spheroidal shapes. They used a good deal of analytical intuition to obtain the corresponding metrics without solving the non-linear potential equation. These and other electro-gravitational solutions are reviewed in the recent work by Ivanov [13.

The Gürses method uses the power of linearity for the construction of sources for MP spacetimes. However, new singularities can be included in the geometry when the parameters take arbitrary values. This fact is simply illustrated by his spherically symmetric solution, which is prone to develope periodic singularities.

This paper is organised as follows: in the next Section we consider fundamental facts of the MP approach and review the proof that the relationship between the electrostatic potential and $V$ is actually a consequence of the field equations. This is followed by a discussion of the electrovac MP solution in the spherically symmetric case. Section III looks at the Gürses asymptotically flat, class $C^{1}$ geometry. We define new parameters that allow a careful choice of families of non-singular solutions. Unbounded dust sources are examined in Section IV, where solutions of the non-linear potential equation are matched to the Gürses internal geometry. In Section V we investigate MP sources with non-Noetherian symmetries. These symmetries are formulated in terms of Lie derivatives of the metric and energy-momentum tensors, and provide us with solutions obtained in a very elegant manner. This approach dispenses with the need for $a d-h o c$ fuctionals $\rho=\rho(V)$ and confronts us with distinctive classes of MP solutions. We conclude in Section VI with a brief discussion of our results. 


\section{Majumdar-Papapetrou Solutions}

We consider solutions for the Einstein-Maxwell-charged dust (EMCD) equations

$$
\begin{gathered}
G_{\mu \nu}=8 \pi T_{\mu \nu}, \\
F_{; \nu}^{\mu \nu}=4 \pi J^{\mu},
\end{gathered}
$$

where $G_{\mu \nu}$ and $F^{\mu \nu}$ denote the Einstein and Maxwell tensors, and the total energymomentum tensor is given by

$$
T_{\mu \nu}=E_{\mu \nu}+M_{\mu \nu} .
$$

The Maxwell energy-momentum tensor is

$$
E_{\mu \nu}=\frac{1}{4 \pi}\left(F_{\mu \alpha} F_{\nu}^{\alpha}-\frac{1}{4} g_{\mu \nu} F_{\alpha \beta} F^{\alpha \beta}\right),
$$

and the matter term

$$
M_{\mu \nu}=\rho u_{\mu} u_{\nu}
$$

corresponds to dust with energy density $\rho$ and four-velocity $u^{\mu}$. The four-current is defined by the expression

$$
J^{\mu}=\sigma u^{\mu},
$$

where $\sigma$ is the charge density.

We assume a static spacetime and use the conformastatic metric

$$
d s^{2}=-V^{2} d t^{2}+\frac{1}{V^{2}} h_{i j} d x^{i} d x^{j},
$$

where the background metric $h_{i j}$ and $V$ depend only on the space-like coordinates $x^{1}$, $x^{2}, x^{3}$. The electrostatic forms of $A_{\mu}$ and $J^{\mu}$ are given by

$$
\begin{gathered}
A_{\mu}=A_{0}\left(x^{i}\right) \delta_{\mu}^{0}, \\
J^{\mu}=\frac{\sigma\left(x^{i}\right)}{V} \delta_{0}^{\mu},
\end{gathered}
$$

with $i=1,2,3$. The corresponding expression for $u^{\mu}$ is

$$
u^{\mu}=\frac{1}{V} \delta_{0}^{\mu},
$$

which clearly satisfies the constraint $u^{\mu} u_{\mu}=-1$.

Using the definition of the Maxwell tensor

$$
F_{\mu \nu}=\partial_{\mu} A_{\nu}-\partial_{\nu} A_{\mu}
$$

and Eqs. (10)-(12), we see that Eq. (5) contains only one non-trivial equation:

$$
\frac{1}{\sqrt{h}} \partial_{j}\left(\sqrt{h} h^{j k} \frac{\partial_{k} A_{0}}{V^{2}}\right)=\frac{4 \pi \sigma}{V^{3}},
$$


where $h$ and $h^{i j}$ are the determinant and the inverse of $h_{i j}$, respectively.

Using Eq. (10) we determine the components of the Einstein tensor:

$$
\begin{gathered}
G_{00}=-3 V^{2} h^{i j} \partial_{i} V \partial_{j} V+2 V^{3} \nabla_{(h)}^{2} V+\frac{1}{2} V^{4} R_{(h)}, \\
G_{0 i}=0 \\
G_{i j}=R_{(h) i j}-\frac{2}{V^{2}} \partial_{i} V \partial_{j} V+h_{i j}\left(\frac{1}{V^{2}} h^{m k} \partial_{m} V \partial_{k} V-\frac{1}{2} R_{(h)}\right),
\end{gathered}
$$

where $\nabla_{(h)}^{2}, R_{(h)}$ and $R_{(h) i j}$ denote the Laplacian operator, the Ricci scalar and the Ricci tensor associated to the background space with metric $h_{i j}$.

A simple procedure yields the components $E_{\mu \nu}$ and $M_{\mu \nu}$ in this static case. The resulting expressions are given by

$$
\begin{gathered}
E_{00}=\frac{1}{8 \pi} V^{2} h^{i j} \partial_{i} A_{0} \partial_{j} A_{0}, \\
E_{0 i}=0 \\
E_{i j}=\frac{1}{4 \pi}\left(-\frac{1}{V^{2}} \partial_{i} A_{0} \partial_{j} A_{0}+\frac{1}{2} \frac{1}{V^{2}} h_{i j} h^{k l} \partial_{k} A_{0} \partial_{l} A_{0}\right),
\end{gathered}
$$

and

$$
M_{00}=\rho V^{2}, \quad M_{0 i}=0, \quad M_{i j}=0 .
$$

The above results allow the explicit determination of Eqs. (身). Assuming that the background space is Euclidean, we obtain the seven non-trivial equations

$$
\begin{gathered}
-3 V^{2} h^{i j} \partial_{i} V \partial_{j} V+2 V^{3} \nabla_{(h)}^{2} V=V^{2} h^{i j} \partial_{i} A_{0} \partial_{j} A_{0}+8 \pi \rho V^{2}, \\
-2 \partial_{i} V \partial_{j} V+h_{i j} h^{k l} \partial_{k} V \partial_{l} V=-2 \partial_{i} A_{0} \partial_{j} A_{0}+h_{i j} h^{k l} \partial_{k} A_{0} \partial_{l} A_{0} .
\end{gathered}
$$

Contracting the last equation with $h^{i j}$ and using $h_{i}^{i}=3$ we find that

$$
h^{k l} \partial_{k} V \partial_{l} V=h^{k l} \partial_{k} A_{0} \partial_{l} A_{0} .
$$

Combining this result with Eq. (24) we get the equation

$$
\partial_{i} V \partial_{j} V=\partial_{i} A_{0} \partial_{j} A_{0}
$$

which can be integrated to obtain

$$
A_{0}=\kappa V, \quad \kappa^{2}=1,
$$

provided we take the additive integration constant equal to zero. This result allows a great simplification of Eq. (23) as well. In fact, using Eq. (27) to eliminate $A_{0}$ in Eq. (23), and combining the resulting expression with the identity

$$
\nabla_{(h)}^{2} V=\frac{2}{V} h^{i j} \partial_{i} V \partial_{j} V-V^{2} \nabla_{(h)}^{2}\left(\frac{1}{V}\right)
$$


we get the Poisson-type equation

$$
\nabla_{(h)}^{2} \lambda+4 \pi \rho \lambda^{3}=0
$$

where $\lambda=\frac{1}{V}$.

We can use Eq. (27) to eliminate $A_{0}$ in the non-trivial Maxwell equation (15) as well. The outcoming expression is

$$
\nabla_{(h)}^{2} \lambda+\frac{4 \pi \sigma}{\kappa} \lambda^{3}=0
$$

Comparing Eqs. (29) and (30) we conclude that $\sigma$ and $\rho$ are related by

$$
\sigma=\kappa \rho .
$$

Equation (31) characterises the MP class of static solutions for the EMCD equations, in which the electrostatic repulsion between charges with the same sign is exactly balanced by the gravitational attraction within the fluid. This kind of fluid has been named electrically counterpoised dust [10]. Such a precise equilibrium of gravity and electricity is physically possible and can be carried out with slightly ionised Hidrogen 11, 14.

We remark that the above analysis of the EMCD equations is a generalisation of the one presented by Lynden-Bell et al. [15], who made the Cartesian choice $h_{i j}=\delta_{i j}$ for the background space metric.

If we assume $\rho=0$, then Eq. (29) reduces to the usual Laplace equation $\nabla_{(h)}^{2} \lambda=$ 0 and the electrovac, multi-black hole solution follows straightforwardly. Assuming spherical symmetry and using spherical coordinates $\left(x^{0}=t, x^{1}=r, x^{2}=\theta, x^{3}=\phi\right)$, we find

$$
\lambda=1+\frac{m}{r}
$$

In the far-asymptotic region, the behaviour of this solution is approximately given by

$$
V \approx 1-\frac{m}{r}, \quad g_{00} \approx-1+\frac{2 m}{r}, \quad A_{0} \approx \pm\left(1-\frac{m}{r}\right) .
$$

The corresponding expression for the electric field is

$$
F_{01} \approx \frac{q}{r^{2}}
$$

where

$$
q=\mp m \text {. }
$$

Equation (10) implies that the invariant area of any 2-sphere surrounding the origin is given by $\frac{4 \pi r^{2}}{V(r)^{2}}$. Therefore, the set $r=0, t=$ constant has a non-zero invariant area given by $4 \pi m^{2}$. In fact, a simple coordinate transform shows that the null hypersurface $r=0$ is the horizon of the extremal Reissner-Nordström solution. Also, if we define the new radial coordinate $\tilde{r}=-r$ and perform the standard analysis [4], then we find that this horizon encloses a point-like, essential singularity placed at $\tilde{r}=m$. In fact, the invariant area vanishes and the scalar $F_{\mu \nu} F^{\mu \nu}=\lambda^{-4}\left(\frac{d \lambda}{d r}\right)^{2}$ blows up at that point. 


\section{The Linear Model}

Equations (29) and (31) were discussed by Das [5] in his study of equilibrium configurations of self-gravitating charged dust. More recently, Gürses [6] has considered non-electrovac solutions when Eq. (29) is linear and homogeneous. This situation corresponds to his choice $\rho=\frac{b^{2}}{4 \pi \lambda^{2}}$ for constant $b$. In this case, Eq. (29) admits the solution

$$
\lambda=\frac{a \sin (b r)}{r},
$$

where $a$ is an integration constant. (The signs of $a$ and $b$ are not relevant to the geometry and we assume $a>0, b>0$.)

The oscillatory behaviour of this solution implies a geometry with complicated radial dependence. In fact, the invariant area vanishes for an infinite set of values of $r$, and the Ricci scalar $R=\frac{2 b^{2} r^{2}}{a^{2} \sin (b r)^{2}}$ blows up wherever the invariant area vanishes, except for $r=0$.

Hartle and Hawking [4] showed that the multi-black hole geometry (2) is the only electrovac solution without nacked singularities. This result motivated Gürses [6] to consider the match of arbitrary electrovac solutions to charged dust solutions, so that the horizons and curvature singularities of the external solutions can be eliminated. Nevertheless, the internal solution must be chosen with care so that new singularities are not included in the geometry. The oscillatory, point-like curvature singularities of the linear model (36) constitute a clear-cut example of this potential trouble.

We develope a new set of parameters to analyse the geometry proposed by Gürses. These parameters allow simple choices for the charged dust solution, so that internal curvature singularities can be avoided.

In order to construct a spherically symmetric, class $C^{1}$ model we require the continuity of the metric and its first radial derivative at the boundary of the dust distribuition. In other words, we want $r \lambda$ and $(r \lambda)^{\prime}$ to be continuous at the coordinate radius $r_{1}>0$ of the charged source. Imposing these matching conditions to the internal and external solutions (36) and (32), we obtain

$$
\begin{gathered}
\tan \beta=(1+\mu) \beta, \\
\alpha=\sqrt{(1+\mu)^{2}+\frac{1}{\beta^{2}}},
\end{gathered}
$$

where

$$
\beta=b r_{1}, \quad \mu=\frac{m}{r_{1}}, \quad \alpha=\frac{a}{r_{1}} .
$$

In terms of the new adimensional parameters, the internal and external solutions are given by

$$
\begin{gathered}
\lambda_{I}=\frac{\alpha \sin (\beta x)}{x}, \quad 0 \leq x \leq 1, \\
\lambda_{I I}=1+\frac{\mu}{x}, \quad 1 \leq x<\infty,
\end{gathered}
$$


where $x=\frac{r}{r_{1}}$. Given a particular value of $\mu>0$, each of the infinite positive roots of Eq. (37) defines a family of asymptotically flat solutions. These solutions are singularityfree only if $\beta<\pi$. Clearly, only one of the positive roots of Eq. (37) satisfies this condition, so that every acceptable family of solutions is defined by some $\beta$ in the interval $\left(0, \frac{\pi}{2}\right)$.

As in the spherically symmetric case considered by Bonnor and Wickramasuriya [11], the linear model implies arbitrarily large redshifts when the mass parameter $\mu$ tends to infinity. Let us consider two spatial points $P$ and $Q$ at rest in the coordinates of the conformastatic metric (10). If we take $Q$ at spatial infinity, the redshift of light emitted at $P$ and received at $Q$ is $\lambda(P)-1$. Using Eq. (40), we find that $\lambda_{I}(r=0)=\alpha \beta$, so that the maximum redshift attainable with this bounded source is

$$
Z=\alpha \beta-1 \text {. }
$$

Using Eqs. (37) and (38), we find that $\beta \approx \frac{\pi}{2}, \alpha \approx \mu$, and $Z$ is unbounded when $\mu$ is arbitrarily large.

\section{The -Sine-Gordon Model}

The non-linear potential equation (29) takes the spherically symmetric form

$$
\frac{d^{2} \lambda}{d r^{2}}+\frac{2}{r} \frac{d \lambda}{d r}+4 \pi \rho \lambda^{3}=0
$$

Using the new radial coordinate $\tau=\frac{1}{r}$, the same differential equation can be written as

$$
\frac{d^{2} \lambda}{d \tau^{2}}+\frac{4 \pi \rho}{\tau^{4}} \lambda^{3}=0
$$

If $\rho$ and $\lambda$ satisfy the condition

$$
\rho=\frac{\delta^{2}}{4 \pi} \frac{\tau^{4} \sin \lambda}{\lambda^{3}}
$$

then (44) finally reduces to the -sine-Gordon equation

$$
\frac{d^{2} \lambda}{d \tau^{2}}+\delta^{2} \sin \lambda=0
$$

It admits the solution

$$
\lambda(\tau)=2 \arcsin [\tanh (\delta \tau+c)],
$$

where $c$ is an integration constant, and $\delta$ is assumed to be positive. In terms of the original radial coordinate, this solution read

$$
V(r)=\frac{1}{2 \arcsin \left[\tanh \left(\frac{\delta}{r}+c\right)\right]} .
$$

We observe that $V(0)^{2}$ is finite. Hence the invariant area vanishes for $r=0$, and the set $r=0, t=$ constant is point-like with respect to this solution. A numerical study 
of the invariants $F_{\mu \nu} F^{\mu \nu}, R, R^{\alpha \beta} R_{\alpha \beta}, R^{\alpha \beta \gamma \delta} R_{\alpha \beta \gamma \delta}, R_{\alpha \beta \gamma \delta} R_{\sigma \rho}^{\gamma \delta} R^{\alpha \beta \sigma \rho}$ indicates that these quantities are bounded for non-negative $r$, whenever $c$ is positive.

If we choose

$$
c=\frac{1}{2} \ln \left[\frac{1+\sin (1 / 2)}{1-\sin (1 / 2)}\right],
$$

then the far-asymptotic $(r \rightarrow \infty)$ behaviour of this solution is given by Eqs. (33)-(35) with $m=2 \delta \cos (1 / 2)$. Therefore, this charged dust solution is asymptotically flat, exactly as the previously considered electrovac solution. Also, Eqs. (47), (49), and (45) imply that the dust energy density of this model is definite positive for $r>0$, provided $m>0$.

The coordinate transform $\tilde{r}=-r$ reveals the existence of a point-like, essential singularity at $\tilde{r}=\frac{\delta}{c}$. In fact, the invariant $F_{\mu \nu} F^{\mu \nu}$ blows up at this point. Additionally, this geometry is asymptotically flat (with negative mass) for $\tilde{r} \rightarrow \infty$.

The above considerations suggest a division of the spacetime manifold into three parts, separated by the point-like singularity placed at $\tilde{r}=\frac{\delta}{c}$ and the time-like worldline $(r=0, t)$. We have seen that two of these parts are asymptotically flat. The third one is defined by $\tilde{r} \in\left(0, \frac{\delta}{c}\right)$ and has spatial volumen $\Omega$ given by the finite integral

$$
\Omega=4 \pi \int_{0}^{\frac{\delta}{c}} \frac{\tilde{r}^{2}}{V(\tilde{r})^{3}} d \tilde{r} .
$$

Although the structure of this charged dust solution is complicated by the juxtaposition of bounded and unbounded spatial regions, we see that the far-asymptotic behaviour is its most relevant feature. In fact, a singularity-free class $C^{1}$ geometry can be built if we match the metric (47) to the Gürses internal solution at $r=r_{1}>0$. The result will be a composite sphere of charged dust which extends to infinity. (In this and the following compound source models, the sign of $\sigma$ is assumed to be the same everywhere.)

The adimensional form of the new solution is given by

$$
\begin{gathered}
\lambda_{I}=\frac{\alpha \sin (\beta x)}{x}, \quad 0 \leq x \leq 1, \\
\lambda_{I I}=2 \arcsin \left[\tanh \left(\frac{\gamma \mu}{x}+c\right)\right], \quad 1 \leq x<\infty,
\end{gathered}
$$

where $\gamma=\frac{1}{2 \cos (1 / 2)}$. In this case, the $C^{1}$ match of $\lambda_{I}$ and $\lambda_{I I}$ implies the equations

$$
\begin{gathered}
\tan \beta=f(\mu) \beta, \\
f(\mu)=\frac{1}{1-\frac{\gamma \mu \sqrt{1-\chi^{2}}}{\arcsin \chi}},
\end{gathered}
$$

and

$$
\alpha=2 \sqrt{\arcsin (\chi)^{2}+\frac{1}{\beta^{2}}\left[\arcsin \chi-\gamma \mu \sqrt{1-\chi^{2}}\right]^{2}},
$$


where $\chi=\tanh (\gamma \mu+c)$.

As in the linear model, each value of the mass parameter $\mu>0$ determines an infinite set of positive roots for equation (53). Once again, only the root satisfying $0<\beta<\frac{\pi}{2}$ defines a family of singularity-free, asymptotically flat solutions. This family is completely fixed when we evaluate $\alpha$ with Eq. (55).

The determination of $\beta$ and $\alpha$ follows essentially the same procedure in the linear and -sine-Gordon models. Nevertheless, the implicitly defined function $\beta(\mu)$ is remarkably different in these two cases. We see that the coefficient of $\beta$ in the right-hand side of Eq. (37) is a linear function of $\mu$. On the other hand, Eq. (54) reveals a totally different structure in $f(\mu)$, which turns out to be bounded for $\mu$ in the interval $[0, \infty)$.

The greatest redshift obtainable from a -sine-Gordon solution can be easily found in the limit of arbitrarily large $\mu$. The definition of $\chi$ and Eq. (55) allow the approxi-

mations $\chi \approx 1, \sqrt{1-\chi^{2}} \approx 2 e^{-(\gamma \mu+c)}$, and $\alpha \beta \approx \pi \sqrt{1+\beta^{2}}$ for large $\mu$. Equations (53) and (54) imply that $\beta$ tends to zero, and the maximum redshift falls to

$$
Z=\pi-1=2.1415 \ldots
$$

in this limit.

\section{Sources with Non-Noetherian Symmetries}

We have seen that Gürses assumed the equation $\rho=\frac{b^{2}}{4 \pi \lambda^{2}}$ to discuss the linear, homogeneous case of the Poisson-type equation (29). Analogously, Varela [7] considered other choices for $\rho$, leading to non-linear Poisson-type equations which are well known in Soliton Physics. The choice $\rho=\frac{\delta^{2} \tau^{4}}{4 \pi} \frac{\sin \lambda}{\lambda^{3}}$ is the starting point for the -sine-Gordon model developed in the previous Section. This $a d-h o c$ functional relationship between $\rho$ and the metric function $\lambda$ is justified only by the interest of the analytic integral of Eq. (29), given by Eq. (47), and the associated spacetime geometry.

A completely different method for constructing sources for Majumdar-Papapetrou spacetimes comes out when we assume the symmetry

$$
£_{\xi} g_{\mu \nu}=\psi g_{\mu \nu}
$$

where the left-hand side is the Lie derivative of the metric along the congruence generated by the vector field $\xi$, and $\psi$ is an arbitrary function of $x^{\alpha}$. Using the conformastatic metric (1) with $V=V(r)$, and choosing

$$
\xi=\xi^{0} \frac{\partial}{\partial t}+\xi^{1} \frac{\partial}{\partial r}
$$

with $\xi^{0}=\xi^{0}(r)$ and $\xi^{1}=\xi^{1}(r)$, we get the following system of equations:

$$
\begin{gathered}
\frac{\partial V}{\partial r} \xi^{1}=\frac{\psi}{2} V \\
V^{2} \frac{\partial \xi^{0}}{\partial r}=0,
\end{gathered}
$$




$$
\begin{aligned}
-\frac{\partial V}{\partial r} \xi^{1}+\frac{\partial \xi^{1}}{\partial r} V & =\frac{\psi}{2} V \\
\left(-r \frac{\partial V}{\partial r}+V\right) \xi^{1} & =r \frac{\psi}{2} V
\end{aligned}
$$

Solving Eqs. (59)-(62), we find that $\xi^{0}$ and $\psi$ are constants, and

$$
\begin{gathered}
\xi^{1}=\psi r, \\
V=D \sqrt{r},
\end{gathered}
$$

where $D$ is another integration constant. This homothetic vector field is naturally extended to

$$
\xi=v+\psi r \frac{\partial}{\partial r}
$$

where $v$ is any linear combination -with constant coefficients- of $\frac{\partial}{\partial t}$ and the three generators of spatial rotations.

The positive definite energy density of this homothetic charged dust sphere can be easily obtained if we combine Eqs. (29) and (64):

$$
\rho=\frac{D^{2}}{16 \pi r}=\frac{D^{4} \lambda^{2}}{16 \pi} .
$$

The charge density is derived from Eq. (31).

This family of solutions describes homothetic charged dust with a point-like curvature singularity at $r=0$. It was originally discussed by Herrera and Ponce de León [16], who used the most general static, spherically symmetric line element expressed in Schwarzschild coordinates. In fact, these authors obtained it as a member of a class of solutions of the Einstein-Maxwell equations admitting conformal motions. What we find out is that the less general conformastatic line element (1) fits the geometrical symmetry (57) uniquely, i.e., the combination of these two equations singles out a particular family of sources with metric (64), and definite positive density (66).

We can use this homothetic dust solution to construct an asymptotically flat, singularity-free, MP spacetime. In order to eliminate the singularity at $r=0$, we match the homothetic and Gürses geometries at $r=r_{1}>0$. Then the homothetic and electrovac solutions are matched across the surface $r=r_{2}$, with $r_{2}>r_{1}$. As in the -sine-Gordon model, this source is made up of two different types of charged dust. We point out that Herrera and Ponce de León matched the homothetic geometry to the interior Schwarzschild solution. This different choice led them to a source with a core composed of a neutral perfect fluid with non-vanishing pressure.

The $C^{1}$ match of metrics $(36)$ and $(64)$ at $r=r_{1}$, and metrics (64) and (32) at $r=r_{2}$ provides us with four conditions, i.e.,

$$
\begin{gathered}
D=\frac{1}{2 \sqrt{r_{2}}}, \\
m=r_{2}, \\
a \sin \left(b r_{1}\right)=2 \sqrt{r_{1} r_{2}},
\end{gathered}
$$




$$
a b \cos \left(b r_{1}\right)=\sqrt{\frac{r_{2}}{r_{1}}} .
$$

We observe that the total mass of this solution equals the radius of the composite dust sphere in these coordinates. However, the ratio of $m$ to $r_{1}$ can be used to label the arising families of geometries. This fact stablishes an interesting link with the linear and -sine-Gordon models, in which $\mu=\frac{m}{r_{1}}$ is the fundamental parameter. Using the parameters $\alpha$ and $\beta$ defined in Eq. (39), and the independent variable $y=\frac{r}{r_{2}}$, we obtain the adimensional form of this solution:

$$
\begin{gathered}
\lambda_{I}=\frac{\alpha \sin (\beta \mu y)}{\mu y}, \quad 0 \leq y \leq \frac{1}{\mu}, \\
\lambda_{I I}=\frac{2}{\sqrt{y}}, \quad \frac{1}{\mu} \leq y \leq 1, \\
\lambda_{I I I}=1+\frac{1}{y}, \quad 1 \leq y<\infty .
\end{gathered}
$$

We choose $\beta$ as the root of $\tan \beta=2 \beta$ in the interval $\left(0, \frac{\pi}{2}\right)$, and note that $\beta$ does not depend on $\mu$ in this case. The remaining parameter $\alpha$ is calculated with the expression

$$
\alpha=\sqrt{\mu\left(4+\frac{1}{\beta^{2}}\right)} .
$$

The simplicity of this method for constructing homothetic MP spacetimes encourages us to study other non-Noetherian symmetries. Our attention focuses on the coupling of charged matter with geometry, and we expect the higher symmetries of the Ricci and energy-momentum tensors to play an important role in the conformation of the sources.

Ricci collineations (RCs) [17],

$$
£_{\eta} R_{\beta \gamma}=0,
$$

are interesting as the Ricci tensor is the trace of the curvature tensor, which is derived from the connection. Therefore the study of RCs has a natural geometrical significance [18.

On the other hand, the energy-momentum tensor represents the external sources of gravity and its symmetries seem more relevant from the physical point of view. Thus the study of matter collineations (MCs),

$$
£_{\zeta} T_{\beta \gamma}=0
$$

should be pertinent to the construction of charged sources.

Studies of this symmetry have been carried out in various contexts. Sharif [19] has looked at MCs in Bianchi and Kantowski-Sachs spacetimes, Carot et al. [20] have considered its algebraic properties in the cases of degenerate and non-degenerate 
energy-momentum tensor, and Hall et al. [18] have treated the similarities between MCs and RCs within a more general framework.

We point out that MCs can be derived from $£_{\zeta} G_{\beta \gamma}=0$ instead of Eq. (76). Obviously, the equivalence of these two equations is guaranteed by Eq. (4).

The analysis of MCs is surprisingly simple when we assume Eq. (1) with $V=$ $V(r)$. The homothetic vector (65) turns out to be a MC when $V=D \sqrt{r}$. Due to its relationship with a lower level symmetry, this MC is said to be improper. Other solutions of Eq. (76) can be found when $\zeta$ takes the form

$$
\zeta=\zeta^{0}(t, r) \frac{\partial}{\partial t}+\zeta^{1}(t, r) \frac{\partial}{\partial r}
$$

In this case, Eq. (76) entails the following system of partial differential equations:

$$
\begin{aligned}
& \frac{\partial Q}{\partial r} \zeta^{1}+2 Q \frac{\partial \zeta^{0}}{\partial t}=0 \\
& P \frac{\partial \zeta^{1}}{\partial t}+Q \frac{\partial \zeta^{0}}{\partial r}=0 \\
& \frac{\partial P}{\partial r} \zeta^{1}+2 P \frac{\partial \zeta^{1}}{\partial r}=0 \\
& \left(2 P+r \frac{\partial P}{\partial r}\right) \zeta^{1}=0
\end{aligned}
$$

where

$$
\begin{gathered}
P=\frac{1}{V^{2}}\left(\frac{\partial V}{\partial r}\right)^{2} \\
Q=\frac{V^{2}}{r}\left[3 r\left(\frac{\partial V}{\partial r}\right)^{2}-2 r V \frac{\partial^{2} V}{\partial r^{2}}-4 V \frac{\partial V}{\partial r}\right] .
\end{gathered}
$$

Assuming $\zeta^{1} \neq 0$, Eqs. (81) and (82) can be solved to obtain

$$
V=H r^{\epsilon},
$$

where $H$ and $\epsilon$ are integration constants. Combined with Eq. (29), this result yields the charged dust energy density

$$
\rho=\frac{\epsilon(1-\epsilon) H^{2}}{4 \pi r^{2(1-\epsilon)}}=\frac{\epsilon(1-\epsilon) H^{\frac{2}{\epsilon}} \lambda^{2\left(\frac{1}{\epsilon}-1\right)}}{4 \pi},
$$

which is definite positive only if $\epsilon \in(0,1)$. Using Eqs. (82)-(84), we calculate $P$ and $Q$ and proceed to solve Eq. (80) for $\zeta^{1}$ and then Eqs. (78) and (79) for $\zeta^{0}$. The straightforward evaluation of the arising integration functions provides us with the results

$$
\zeta=\frac{\ln r}{3 H^{4}} \frac{\partial}{\partial t}+r t \frac{\partial}{\partial r}
$$

for $\epsilon=\frac{1}{2}$, and

$$
\zeta=\left[\frac{\epsilon}{H^{4}(\epsilon-2)(4 \epsilon-2) r^{(4 \epsilon-2)}}+\left(\frac{1}{2}-\epsilon\right) t^{2}\right] \frac{\partial}{\partial t}+r t \frac{\partial}{\partial r}
$$


otherwise.

The Kretschmann scalar derived from (84) is given by

$$
R^{\alpha \beta \gamma \delta} R_{\alpha \beta \gamma \delta}=\frac{4 H^{4} \epsilon^{2}\left(7 \epsilon^{2}-12 \epsilon+7\right)}{r^{4(1-\epsilon)}},
$$

and the invariant area of any 2-sphere which is inmerse in the fluid and sorrounds the origin of coordinates is $\frac{4 \pi r^{2(1-\epsilon)}}{H^{2}}$. Thus the point-like curvature singularity at $r=0$ exists for every $\epsilon \in(0,1)$.

We match our MC solution (84) to the Gürses and electrovac geometries at $r=r_{1}$ and $r=r_{2}>r_{1}$, respectively. The arising class $C^{1}$, asymptotically flat solution is given by

$$
\begin{gathered}
\lambda_{I}=\frac{\alpha \sin \left[\left(\frac{1}{\epsilon}-1\right) \beta \mu y\right]}{\left(\frac{1}{\epsilon}-1\right) \mu y}, \quad 0 \leq y \leq \frac{1}{\left(\frac{1}{\epsilon}-1\right) \mu}, \\
\lambda_{I I}=\frac{1}{(1-\epsilon) y^{\epsilon}}, \quad \frac{1}{\left(\frac{1}{\epsilon}-1\right) \mu} \leq y \leq 1, \\
\lambda_{I I I}=1+\frac{\epsilon}{(1-\epsilon) y}, \quad 1 \leq y<\infty,
\end{gathered}
$$

where

$$
\alpha=\left(\frac{1}{\epsilon}-1\right)^{\epsilon} \mu^{\epsilon} \sqrt{\frac{1}{(1-\epsilon)^{2}}+\frac{1}{\beta^{2}}} .
$$

The total mass and the radius of the composite dust sphere are related by

$$
\frac{m}{r_{2}}=\frac{\epsilon}{1-\epsilon}
$$

This class of solutions is singularity-free whenever $\beta$ is chosen as the root of

$$
\tan \beta=\frac{\beta}{1-\epsilon}
$$

within the interval $\left(0, \frac{\pi}{2}\right)$ for $\epsilon \in(0,1)$.

The study of RCs in MP spacetimes has not been carried out very far; however, some points have been noted. A similar method can be used to solve Eq. (75) and construct charged dust sources admitting RCs with $(t, r)$ dependence. The system of equations corresponding to Eqs. (78)-(81) has a more complicated dependence on $V$ and its derivatives, and a third order differential equation must be solved to find the metric function

$$
V=K r^{\varepsilon} e^{\frac{\omega}{r}}
$$

where $K, \varepsilon$, and $\omega$ are integration constants. The evaluation of integration functions forces us to choose $\omega=0$, so that Eq. (95) actually reduces to the MC metric, given by Eq. (84). 


\section{Final Remarks}

We have constructed several types of sources for the Einstein-Maxwell field in the MP case of the theory. These are static charged dust spheres for which the energy and charge densities satisfy Eq. (31). In all cases, the central region is described by the Gürses solution which is non-singular whenever the values of the parameters are appropiately fixed.

The linear and -sine-Gordon models are asymptotically flat and have the same $r^{-2}$ dependence in the electric field at large distances from the symmetry center. The linear model assumes a bounded charge distribution, and the -sine-Gordon geometry describes charged dust which extends to infinity. This unbounded source becomes dilute at infinity fast enough so that the total mass (and charge) is finite [21].

Although both geometries have the same asymptotic properties, an observer at spatial infinity can discrimininate between them. To this end, the observer must measure the maximum redshift attainable from these sources for very large values of $\mu$.

The study of homothetic charged dust sources for MP spacetimes led us to a solution previously found by Herrera and Ponce de León in Schwarzschild coordinates. The simplicity of our calculation in isotropic coordinates pointed out an interesting affinity between this symmetry and the conformastatic metric with $V=V(r)$. This observation motivated the consideration of higher non-Noetherian symmetries in MP spacetimes.

We have solved the MC equations (76) to find a class of MP solutions with positive dust energy density, which includes the homothetic solution as a particular case. The analytical forms of the associated vector fields indicate that the homothetic solution plays a distinctive role within this class. Preliminary calculations suggest that these MCs are proper (not derived from any lower symmetry), but additional work is due in order to clarify this aspect of the solutions.

The above observations encourage us to investigate the whole structure of the MCs in spherically symmetric MP spacetimes. The fact that the energy-momentum tensor is non-degenerate will determine a finite dimensional Lie algebra of MCs [19, 20] associated to the metric (84). A similar study of the corresponding RCs could be carried out in detail and might prove interesting. This future work should convey us to a classification scheme for MP solutions based on non-Noetherian symmetries.

\section{Acknowledgments}

The author acknowledges the use of the MAPLE program and the TENSOR package at various stages of this work. 


\section{References}

[1] S. D. Majumdar, Phys. Rev. 72, 390 (1947).

[2] A. Papapetrou, Proc. R. Irish Acad. A51, 191 (1947).

[3] J. L. Synge, Relativity: the General Theory. North-Holland, Amsterdam, 1960.

[4] J. B. Hartle and S. W. Hawking, Commun. Math. Phys. 26, 87 (1972).

[5] A. Das, Proc. R. Soc. London A267, 1 (1962).

[6] M. Gürses, Phys. Rev. D 58, 044001 (1998).

[7] V. Varela, Analytical Description of Voids in Majumdar-Papapetrou Spacetimes, in the Proceedings of the Spanish Relativity Meeting, ERE-99, Bilbao, España, 1999. Edited by J. Ibañez (Servicio Editorial de la Universidad del País Vasco, 2000). Preprint gr-qc/9911062.

[8] D. Ida, Prog. Theor. Phys. 103, 573 (2000).

[9] Y. Cho, Y. Degura and K. Shiraishi, Phys. Rev. D 62, 084038 (2000).

[10] W.B. Bonnor and S.B.P. Wickramasuriya, Int. J. Theor. Phys. 5, 371 (1972).

[11] W.B. Bonnor and S.B.P. Wickramasuriya, Mon. Not. R. Astr. Soc. 170, 643 (1975).

[12] W.B. Bonnor, Class. Quant. Grav. 15, 351 (1998).

[13] B.V. Ivanov, preprint gr-qc/0109010.

[14] W.B. Bonnor, Class. Quant. Grav. 16, 4125 (1999).

[15] D. Lynden-Bell, J. Bičák and J. Katz, Annals Phys. 271, 1 (1999).

[16] L. Herrera and J. Ponce de León, J. Math. Phys. 26, 2302 (1985).

[17] A.H. Bokhari and A. Qadir, J. Math. Phys. 34, 3543 (1993); M.J. Amir, A.H. Bokhari and A. Qadir, J. Math. Phys. 35, 3005 (1994); R. Bertolotti, G. Contreras, L.A. Núñez, U. Percoco and J. Carot, J. Math. Phys. 37, 1086 (1996); J. Carot, L.A. Núñez and U. Percoco, Gen. Rel. Grav. 29, 1223 (1997); M. Tsamparlis and P.S. Apostolopoulos, Gen. Rel. Grav. 32, 281 (2000); G. Contreras, L.A. Núñez and U. Percoco, Gen. Rel. Grav. 32, 285 (2000).

[18] G.S. Hall, I. Roy and E.G.L.R. Vaz, Gen. Rel. Grav. 28, 299 (1996).

[19] M. Sharif, preprint gr-qc/0101124.

[20] J. Carot, J. da Costa and E.G.L.R. Vaz, J. Math. Phys. 35, 4832 (1994)

[21] The asymptotic behaviour of unbounded, neutral fluid sources with finite mass has been recently studied in R. Beig and M. Karadi, Class. Quant. Grav. 18, 671 (2001). 